\title{
ARGUMENT ON THE THEME
}

Sorinel Mocanu ${ }^{2}$

Even without being a truly psychoanalytic object (in Bionian terms), the secret can be articulated with many elements of psychoanalytic theory and practice.

Implied from the start by the psychoanalytic frame (a secure place hidden from the others and from where nothing can 'escape' without patient's accord), or by the analyst's 'neutral 'attitude (allowing secrets to be kept as long as needed for the patient and to be revealed in time as 'gifts' for the analyst or as revelations for himself), the secret has a structuring function in setting out boundaries between interior and exterior, while also enabling a personal place/space involved in creating personal identity.

Provocative by the curiosity stirred, and - sometimes - by the negative capacity required to endure the other's secret, this topic is also a very actual subject in a world of dwindling privacy and increasing connectivity more than ever. That is testing our confidence in the other and our capacity to bear the loneliness that is sometimes created.

Not having the capacity to hide, so everything is out - that is one of the features of a paranoid patient, contrasting with the many taboos of the secretive one. Both are impinging on communication in such a way that sometimes the content can be revealed only by an 'architecture of the negative' - what is the patient not talking about?

Quoting Winnicott, we invite you to allow this issue to hide itself, but also to discover some of its secrets. 